\title{
Escalas para medir necesidades de cuidado en pacientes, familia y profesionales que se enfrentan al final de la vida en la UCI
}

\author{
Scales to measure care needs in patients, family and \\ professionals facing the end of life in the UCI
}

Escalas para medir as necessidades de atendimento em pacientes, famílias e profissionais que enfrentam o fim da vida na UCI

\begin{abstract}
Milena Hernandez-Zambrano ${ }^{1}$, Ana Julia Carrillo-Algarra ${ }^{2}$, Lady
Bibiana Torres-Pachon ${ }^{3}$, Dely Marcela Herrera-Cristancho ${ }^{4}$

${ }^{1}$ Enfermera, PhD. en Ciencias de la Salud, Mg. en cuidados de salud para la promoción de la autonomía de las personas y la atención a los procesos de fin de vida. Docente Investigadora, Facultad de Enfermería. Fundación Universitaria de Ciencias de la Salud. Bogotá, Colombia. Correo electrónico:

smhernandez3@fucsalud.edu.co

${ }^{2}$ Enfermera, Mg. en Administración en Salud. Docente, Facultad de

Enfermería. Fundación Universitaria de Ciencias de la Salud. Bogotá,

Colombia. Correo electrónico: ajcarrillo@fucsalud.edu.co

${ }^{3}$ Enfermera, Especialista de Enfermería en Cuidado Crítico. Facultad de

Enfermería. Fundación Universitaria de Ciencias de la Salud. Bogotá,

Colombia. Correo electrónico: lbtorres1@fucsalud.edu.co

${ }^{4}$ Enfermera, Especialista de Enfermería en Cuidado Crítico. Facultad de

Enfermería. Fundación Universitaria de Ciencias de la Salud. Bogotá,

Colombia. Correo electrónico: dmherrera1@fucsalud.edu.co
\end{abstract}

Cómo citar este artículo en edición digital: Hernandez-Zambrano, S. M., Carrillo-Algarra, A. J., Torres-

Pachon, L. B., E Herrera-Cristancho, D. M. (2019). Escalas para medir necesidades de cuidado en pacientes, familia y profesionales que se enfrentan al final de la vida en la UCI. Cultura de los Cuidados (Edición digital), 23(54). Recuperado de http://dx.doi.org/10.14198/cuid.2019.54.30

Correspondencia: Cra. 19 No. 8A-32, Fundación Universitaria de Ciencias de la Salud. Bogotá, Colombia. Teléfono / fax: +(571) 4375401

Correo electrónico de contacto: smhernandez3@fucsalud.edu.co Recibido: 15/11/2018; Aceptado: 03/03/2019

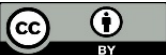

\section{ABSTRACT}

Introduction: The needs generated at the end of life can influence not only the physical health but also the emotional, psychological and spiritual health of those who coexist in a critical care environment.

Objective: To describe the scales that allow to determine the needs of care in patients, family and professionals that face the end of life in the ICU.

Method: Integrative review of the literature. A search was conducted in the databases: PubMed, CINAHL, CUIDEN, Ovid, Epistemonikos, BVS, EBMR, 
repositories and cross-reference analysis.

Results: After the process of critical reading, 13 articles met the inclusion criteria: 4 psychometric validations, 2 systematic reviews and 7 descriptive quantitative studies. The results were classified into three categories: measurement instruments for patients, family and professionals.

Discussion: It was found within the category instruments of measurement of needs focused on the family 27 scales, the best known the CCFNI. For patients, a great research trajectory of needs measurement was identified using the CARE-Q scale. As for UCI professionals, there is no evidence of a scale that measures needs beyond those of work.

Keywords: Measurement instruments, needs, patients, family, professionals, hospice care.

\section{RESUMO}

Introdução: As necessidades que são gerados no final da vida, pode influenciar não só a saúde física, mas no emocional, psicológico e espiritual daqueles que vivem em um ambiente de cuidados intensivos.

Objetivo: Descrever as escalas para determinar as necessidades de cuidados de pacientes, familiares e profissionais que enfrentam o fim da vida na UTI.

Método: Revisão integrativa da literatura. A pesquisa foi realizada nas bases de dados: PubMed, CINAHL, CUIDEN, Ovid, Epistemonikos, BVS, EBMR, repositórios e de análise de referência cruzada.

Resultados: Após o processo de leitura crítica, 13 artigos atenderam aos critérios de inclusão: 4 validações psicométricas, 2 revisões sistemáticas e 7 estudos descritivos quantitativos. Os resultados foram classificados em três categorias: instrumentos de medida para pacientes, familiares e profissionais.

Discussão: Constatou-se dentro da categoria instrumentos de mensuração das necessidades focadas na família 27 escalas, as mais conhecidas do CCFNI. Para os pacientes, uma grande trajetória de pesquisa de mensurações de necessidades foi identificada com a escala CARE-Q. Quanto aos profissionais da UCI, não há evidência de uma escala que mensure necessidades além daquelas do trabalho.

Palavras chave: Instrumentos de medida, necessidades, pacientes, familiares, profissionais, cuidados paliativos na terminalida de da vida.

\section{RESUMEN}

Introducción: Las necesidades que se generan al final de la vida, pueden influenciar no solo la salud física sino en la emocional, psicológica y espiritual de quienes conviven en un entorno de cuidado crítico.

Objetivo: Describir las escalas que permitan determinar las necesidades de cuidado en pacientes, familia y profesionales que se enfrentan al final de la vida en la UCI.

Método: Revisión integradora de la literatura. Se realizó una búsqueda en las bases de datos: PubMed, CINAHL, CUIDEN, Ovid, Epistemonikos, BVS, EBMR, repositorios y análisis de referencias cruzadas.

Resultados: Tras el proceso realizado de lectura crítica 13 artículos cumplían con criterios de inclusión: 4 validaciones psicométricas, 2 revisiones sistemáticas y 7 estudios cuantitativos descriptivos. Los resultados se clasificaron en tres categorías: instrumentos de medición para pacientes, familia y profesionales.

Discusión: Se encontró dentro de la 
categoría instrumentos de medición de necesidades enfocada a la familia 27 escalas, la más conocida la CCFNI. Para pacientes se identificó una gran trayectoria investigativa de medición de necesidades utilizando la escala CARE-Q. En cuanto a los profesionales de la UCI no hay evidencia de una escala que mida las necesidades más allá de las laborales.

Palabras clave: Evaluación de necesidades, pacientes, familia, profesionales, cuidados paliativos al final de la vida.

\section{INTRODUCCIÓN}

Las Unidades de Cuidado Intensivo (UCI), son espacios hospitalarios disponibles para el manejo de personas en condición de salud crítica. Es por ello que la intensidad de la experiencia que supone el paso por una UCI es innegable para todo aquel que interactúa en este medio (Gutiérrez et al., 2008).

En primer lugar, el paciente y la familia, se enfrenta a un entorno desconocido y la cercanía con la muerte les genera incertidumbre, se sienten vulnerables, con dolor físico y espiritual, sentimientos de soledad, depresión y sufrimiento (Enciso, 2015). Según lo reportado en estudios realizados en Estados Unidos y Europa, la tasa de mortalidad en la UCI fluctúo entre el 12,83\% y el 20\% (Ballester et al., 2011; Sprung et al. 2003; Marin et al., 2016) y el índice de supervivencia osciló entre $6.4 \%$ a $40 \%$, variación atribuible a las características de los pacientes y las patologías por las cuales son ingresados (Bagshaw et al., 2009).

Por otra parte, para los profesionales de la UCI que conviven con la muerte de manera cotidiana, el afrontamiento del sufrimiento y la toma de decisiones al final de la vida constituye una de las tareas más difíciles y estresantes a las que se enfrentan. A pesar de que la muerte es parte su contexto laboral, se pueden despertar sentimientos de impotencia, indiferencia e incluso de evasión y negación en relación a la muerte (Vicensi, 2016) generando en el personal estrés, en gran proporción relacionado con el contacto con el dolor y el sufrimiento (Zambrano, 2008).

Considerando lo expuesto anteriormente, se vienen promoviendo diferentes iniciativas y consensos internacionales en el ámbito de la Medicina Crítica, para mejorar los cuidados al final de la vida en la UCI. Así, por ejemplo, en la 5. a Conferencia Internacional en Medicina Crítica, se defiende la importancia de garantizar la provisión del mejor tratamiento posible a los pacientes moribundos y a sus cuidadores (Breen et al., 2001; Carlet et al., 2004). Sin embargo, promover un clima que favorezca una buena muerte en estas unidades no es fácil, es así que durante la última década ha crecido el interés por estudiar cómo se puede mejorar el cuidado en el proceso del final de la vida en la UCI (Jimeno et al., 2007). En este sentido, el objetivo de este estudio fue describir las escalas que permitan determinar las necesidades de cuidados en pacientes, familia y profesionales que se enfrentan al final de la vida en la UCI.

\section{METODOLOGÍA}

Revisión integradora de la literatura. Se planteó el proceso de búsqueda mediante 3 fases: estrategias de búsqueda y criterios de selección, selección de los estudios y evaluación de calidad.

Estrategia de búsqueda y criterios de selección. Se realizó una búsqueda en las bases de datos PubMed, CINAHL, CUIDEN, Ovid, Epistemonikos, BVS, EBMR. Las ecuaciones de búsqueda y títulos identificados se describen en la tabla 1. Posteriormente se 
revisó literatura gris en repositorios universitarios y motores de búsqueda como: Universidad de la Sabana, GOOGLE ACADEMICO, World Wide Science,
OAISTER Por último, se analizó toda referencia cruzada que aportara información adicional.

\section{TABLA 1: Ecuaciones de búsqueda y títulos identificados}

\begin{tabular}{|c|c|c|}
\hline Base de datos & Ecuación & $\begin{array}{l}\text { Títulos } \\
\text { identificados }\end{array}$ \\
\hline \multirow[t]{6}{*}{ Biblioteca Virtual en Salud } & $\begin{array}{l}\text { Necesidades AND Paciente critico OR Unidades de } \\
\text { cuidados intensivos AND enfermería }\end{array}$ & 288 \\
\hline & $\begin{array}{l}\text { Necesidades And Familia OR Paciente Critico AND } \\
\text { Unidades de cuidados intensivos }\end{array}$ & 15 \\
\hline & $\begin{array}{l}\text { Enfermería AND Paciente critico AND Unidades de } \\
\text { cuidados intensivos AND Medición }\end{array}$ & 27 \\
\hline & $\begin{array}{l}\text { Enfermería AND Cuidado AND Unidades de cuidados } \\
\text { intensivos AND Escala }\end{array}$ & 105 \\
\hline & $\begin{array}{l}\text { Medición AND cuidado AND enfermería AND paciente } \\
\text { critico OR Unidades de cuidados intensivos }\end{array}$ & 38 \\
\hline & Necesidades AND paciente critico AND UCI & 36 \\
\hline CUIDEN Plus & $\begin{array}{l}\text { Enfermería AND Paciente critico AND Unidades de } \\
\text { cuidados intensivos AND Medición }\end{array}$ & 49 \\
\hline \multirow[t]{3}{*}{ Nursing@Ovid } & Needa assessments AND Intensive Care Units AND Nurse & 171 \\
\hline & $\begin{array}{l}\text { Critical Illnesses AND Intensive Care Units AND Nurse } \\
\text { AND scale }\end{array}$ & 100 \\
\hline & $\begin{array}{l}\text { Critical Illnesses OR Families AND Needs Assessments } \\
\text { AND Intensive Care Units }\end{array}$ & 6102 \\
\hline \multirow[t]{3}{*}{ PubMed } & $\begin{array}{l}\text { Critical Illnesses AND Intensive Care Units AND Nurse } \\
\text { AND Scale }\end{array}$ & 99 \\
\hline & $\begin{array}{c}\text { Critical Illnesses OR Families AND Needs Assessments } \\
\text { AND Intensive Care Units }\end{array}$ & 371 \\
\hline & $\begin{array}{l}\text { Critical Illnesses AND Nursing AND Needs Assessments } \\
\text { AND Intensive Care Units }\end{array}$ & 85 \\
\hline \multirow[t]{5}{*}{ CINAHL } & $\begin{array}{c}\text { Needs Assessments AND Intensive Care Units AND Critical } \\
\text { Illnesses OR Nursing }\end{array}$ & 145,998 \\
\hline & $\begin{array}{l}\text { Critical Illnesses OR Families AND Needs Assessments } \\
\text { AND Intensive Care Units }\end{array}$ & 68 \\
\hline & $\begin{array}{c}\text { Needs Assessments AND Intensive Care Units AND Critical } \\
\text { Illnesses OR Nursing }\end{array}$ & 145,998 \\
\hline & $\begin{array}{l}\text { Critical Illnesses AND Intensive Care Units AND Nurse } \\
\text { AND Scale }\end{array}$ & 499 \\
\hline & Needs Assessments AND Critical Illnesses & 262 \\
\hline Evidence - Based Medicine & Needs Assessments AND Critical Illnesses & 7571 \\
\hline
\end{tabular}




\section{Cultura de los Cuidados}

\begin{tabular}{|c|c|c|}
\hline Reviews (EBMR) & $\begin{array}{c}\text { Critical Illnesses OR Families AND Needs Assessments } \\
\text { AND Intensive Care Units }\end{array}$ & 35 \\
\hline Epistemonikos & $\begin{array}{c}\text { Necesidades AND Paciente critico OR Unidades de } \\
\text { cuidados intensivos }\end{array}$ & 1876 \\
\hline & $\begin{array}{c}\text { Enfermería OR Cuidado AND Necesidades AND Unidades } \\
\text { de cuidados intensivos }\end{array}$ \\
\hline & $\begin{array}{c}\text { Critical Illnesses AND Intensive Care Units AND Nurse } \\
\text { AND Scale }\end{array}$ \\
\hline & Necesidades AND Familia OR paciente critico & 61 \\
\hline
\end{tabular}

FUENTE: Elaboración propia

Se establecieron los criterios de inclusión y exclusión (Tabla 2). Se realizó una lectura inicial de los títulos, resúmenes y textos completos que aportaron al objetivo de la revisión. Se recuperaron artículos de investigación primaria y secundaria disponibles en texto completo, localizados desde la plataforma de la Fundación
Universitaria de Ciencias de la salud (FUCS) que permitió acceder a la mayor parte de la muestra bibliográfica por contar con la licencia de las bases de datos seleccionadas para la búsqueda. El filtro de texto completo se realizó tras la lectura de los resúmenes que cumplían con los criterios de inclusión.

\section{TABLA 2: Criterios de inclusión y exclusión}

\begin{tabular}{|c|c|}
\hline Criterios de inclusión & Criterios de exclusión \\
\hline $\begin{array}{c}\text { Estudios con pacientes, familiares y } \\
\text { profesionales de UCI Adulto. }\end{array}$ & UCI Pediátrico, neonatal, maternidade. \\
\hline $\begin{array}{c}\text { Valoración de las necesidades a partir de } \\
\text { escalas, cuestionarios e instrumentos. }\end{array}$ & $\begin{array}{c}\text { Estudios que no utilizaron cuestionarios, } \\
\text { escalas o instrumentos. }\end{array}$ \\
\hline Investigaciones primarias, secundarias. & $\begin{array}{c}\text { Estudios con más de } 10 \text { años de } \\
\text { realización. }\end{array}$ \\
\hline Artículos de texto completo. & \\
\hline
\end{tabular}

FUENTE: Elaboración propia

La estrategia de búsqueda inicial arrojó en total 38.471 artículos, a los cuales se le aplicaron los criterios de inclusión y exclusión. El uso de filtros permitió excluir 36.483 artículos tal como se explica en la figura 1.

Selección de estudios. Se eligieron 103 títulos aptos para responder al objetivo planteado, se analizaron un total de 24 resúmenes de los cuales 13 cumplían con los criterios de selección. De los artículos seleccionados se analizaron datos como: autor, año de publicación, idioma, país, objetivo, metodología, resultados relevantes y conclusiones. 


\section{Cultura de los Cuidados}

Evaluación de calidad. Se realizó mediante la aplicación de las listas de comprobación CASPe, y STROBE para estudios descriptivos observacionales; y para los estudios psicométricos se utilizó la lista de chequeo propuesta que Sánchez \& Echeverry (2004) para evaluar la calidad de la validación de una escala: validez, confiabilidad y utilidad. La lectura se realizó por dos investigadores de forma independiente, en caso de discrepancia, el artículo se evaluó por un tercer investigador. Siendo este estudio una revisión no representó riesgo para las personas, se tuvo en cuenta la resolución 8430 de 1993 para estudios documentales y la ley 44 de 1993 donde se esclarece la responsabilidad del investigador con relación de la protección al derecho de autor de los artículos utilizados en este documento mediante el adecuado referenciación bibliográfica.

\section{FIGURA 1: Selección de artículos*}

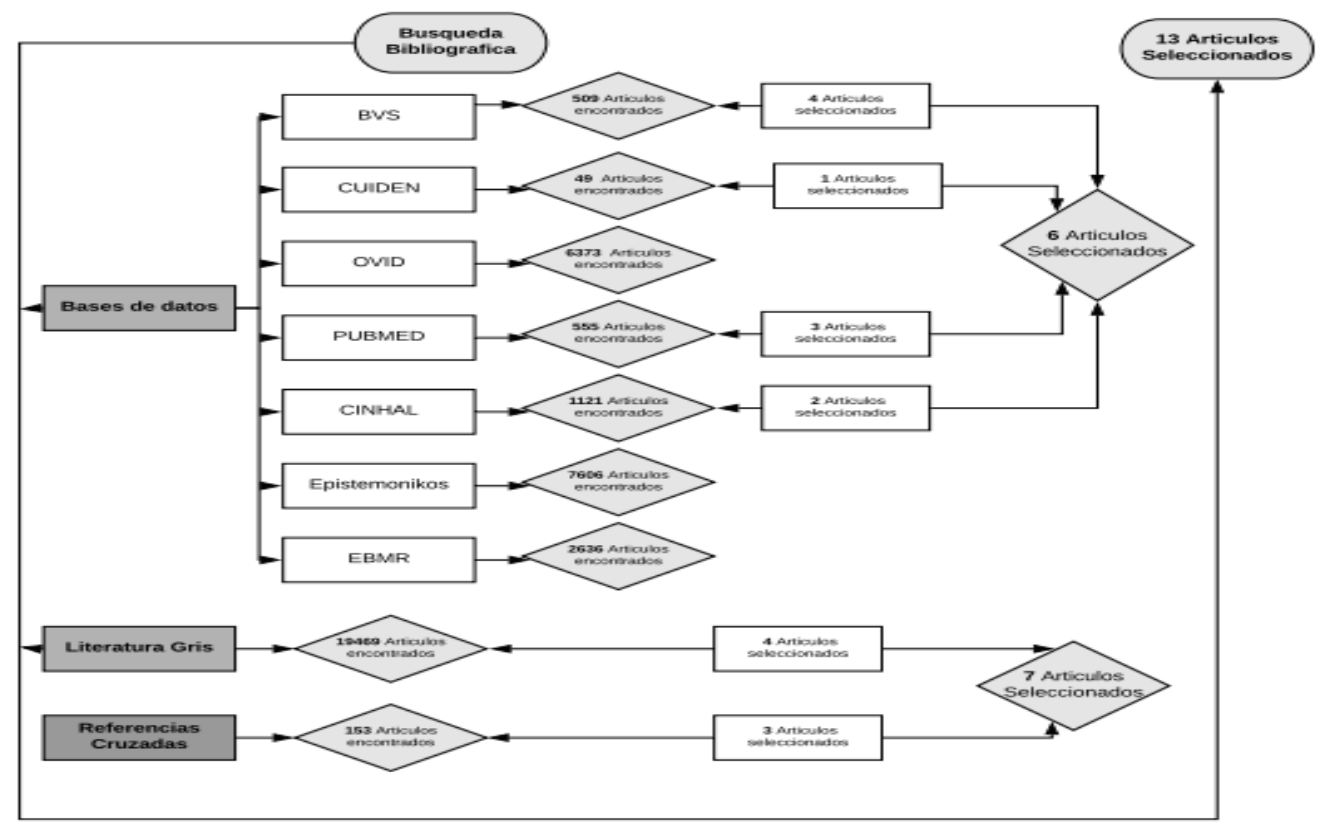

FUENTE: Elaboración propia

*Motivos de exclusión: Población de estudio pediátrica, investigaciones realizadas en contexto diferente a la UCI, estudios que no utilizaron cuestionarios, escalas o instrumentos para medir las necesidades de pacientes, familiares o profesionales. 


\section{RESULTADOS}

Con respecto a los diseños de los estudios incluidos en la revisión, en su mayoría son Descriptivos cuantitativos $(n=7)$, seguidos de estudios Psicométricos $(\mathrm{n}=4)$ y Revisiones Sistemáticas $(\mathrm{n}=2)$; la tabla 3 resume las características de los artículos seleccionados y en la tabla 4 se describen las características de cada escala, resaltando utilidad, validez, ítems, y categorías que mide.

\section{TABLA 3: Resultados de los estudios incluidos en la Revisión Integradora de la Literatura}

\begin{tabular}{|c|c|c|c|c|}
\hline \multicolumn{5}{|c|}{ Categoría instrumentos de medición de necesidades de pacientes } \\
\hline $\begin{array}{c}\text { Autores } \\
\text { y año }\end{array}$ & $\begin{array}{l}\text { Objetivo } \\
\text { principal }\end{array}$ & $\begin{array}{l}\text { Tipo de } \\
\text { estudio }\end{array}$ & $\begin{array}{l}\text { Mediciones } \\
\text { (Cuestionarios } \\
\text { entrevistas) }\end{array}$ & Resultados Relevantes \\
\hline $\begin{array}{l}\text { Pedrechi } \\
\text { \&Camargo } \\
\text { (2015). }\end{array}$ & $\begin{array}{l}\text { Determinar la } \\
\text { percepción de los } \\
\text { comportamientos } \\
\quad \text { en la UCI. }\end{array}$ & $\begin{array}{l}\text { Descriptivo } \\
\text { transversal }\end{array}$ & CARE-Q & $\begin{array}{l}\text { El análisis individual de las categorías, } \\
\text { expresó que la categoría explica y } \\
\text { facilita obtuvo el menor nivel de } \\
\text { percepción por parte de la enfermera. }\end{array}$ \\
\hline $\begin{array}{l}\text { Sepúlveda, } \\
\text { Cárdenas } \\
\text { \& Rojas } \\
\text { (2009). }\end{array}$ & $\begin{array}{c}\text { Establecer } \\
\text { indicadores de } \\
\text { confiabilidad y } \\
\text { validez del } \\
\text { Cuestionario Corto } \\
\text { de Evaluación de la } \\
\text { satisfacción del } \\
\text { usuario acerca del } \\
\text { cuidado de } \\
\text { enfermería en } \\
\text { versión al } \\
\text { español en } \\
\text { población } \\
\text { colombiana. }\end{array}$ & Psicométrico & CARE-Q & $\begin{array}{l}\text { Confiabilidad: alfa: } 0.86 \% \text {, análisis de } \\
\text { validez alfa } 0.627 \% \text {, se facilita alfa de } \\
0.57 \% \text {, analizar su comportamiento en } \\
\text { otras poblaciones. }\end{array}$ \\
\hline $\begin{array}{c}\text { Silva et al. } \\
\text { (2012). }\end{array}$ & $\begin{array}{c}\text { Describir la } \\
\text { experiencia de } \\
\text { construir un } \\
\text { instrumento de } \\
\text { colección de datos } \\
\text { para documentar el } \\
\text { primer paso del } \\
\text { proceso de } \\
\text { enfermería en una } \\
\text { unidad de cuidados } \\
\text { intensivos (UCI). }\end{array}$ & Psicométrico & $\begin{array}{l}\text { Histórico de } \\
\text { enfermería }\end{array}$ & $\begin{array}{l}\text { Creación de instrumento para } \\
\text { documentar y } \\
\text { recolección de datos, validado y } \\
\text { elaborado por enfermeros con } \\
\text { diagramación para el proceso de } \\
\text { enfermería con guía en la teoría de } \\
\text { necesidades } \\
\text { básicas de Horta. }\end{array}$ \\
\hline
\end{tabular}




\begin{tabular}{|c|c|c|c|c|}
\hline $\begin{array}{l}\text { Ramalho } \\
\text { et al. } \\
\text { (2012). }\end{array}$ & $\begin{array}{c}\text { Construir un } \\
\text { instrumento de } \\
\text { levantamiento de } \\
\text { datos de enfermería } \\
\text { para clientes de una } \\
\text { UTI General }\end{array}$ & Psicométrico & $\begin{array}{l}\text { Cuestionario de } \\
\text { necesidades } \\
\text { humanas básicas }\end{array}$ & $\begin{array}{c}\text { IC }>0.80 \text { habla del predominio de las } \\
\text { necesidades humanas básicas } \\
\text { afectadas en el adulto críticamente } \\
\text { enfermo. }\end{array}$ \\
\hline $\begin{array}{l}\text { Olano \& } \\
\text { Vivar } \\
\text { (2012). }\end{array}$ & $\begin{array}{c}\text { Conocer y } \\
\text { examinar las } \\
\text { características de } \\
\text { instrumentos } \\
\text { válidos para } \\
\text { valorar las } \\
\text { necesidades de los } \\
\text { familiares de UCI }\end{array}$ & $\begin{array}{l}\text { Revisión } \\
\text { sistemática }\end{array}$ & $\begin{array}{l}\text { CCFNI, FS-ICU, } \\
\text { SCCMFNA, } \\
\text { FAMILY } \\
\text { SATISFACTION } \\
\text { SURVEY, CCFSS }\end{array}$ & $\begin{array}{l}\text { CCFNI (45 ítems) es el mas utilizado } \\
\text { desde su } \\
\text { creación, escala tipo Likert 1-4, } \\
\text { estabilidad de } 64,71 \text { - } 96,08 \% \text {, validez } \\
\text { alfa: 0,61 - 0,88. FS-ICU ( } 34 \text { ítems) } \\
\text { escala de puntuación y pregunta } \\
\text { cerrada, fiabilidad 0,847, validez: alfa: } \\
\text { 0.74- 0,97\%. SCCMFNA (14 } \\
\text { ítems) escala de puntuación, fiabilidad } \\
\text { confiada sin } \\
\text { registros, valides: alfa } 0.76 \% \text {. FAMILY } \\
\text { (15 ítems): }\end{array}$ \\
\hline $\begin{array}{l}\text { Gómez, } \\
\text { Ballester \& } \\
\text { Gil (2011). }\end{array}$ & $\begin{array}{c}\text { Identificar las } \\
\text { necesidades de los } \\
\text { familiares de los } \\
\text { pacientes } \\
\text { hospitalizados en la } \\
\text { unidad de cuidado } \\
\text { intensivo. }\end{array}$ & Psicométrico & CCFNI & $\begin{array}{l}\text { Alfa: } 0,65 \% \text {, validez de constructo se } \\
\text { eliminaron } 3 \text { ítems } 8,12,13 \text {, prueba } \\
\text { KMO obtuvo un índice } 0,60 \text {, tiene un a } \\
\text { adecuada bondad psicométrica en la } \\
\text { población española. }\end{array}$ \\
\hline $\begin{array}{c}\text { Ban, et al. } \\
\text { (2015). }\end{array}$ & $\begin{array}{c}\text { Determinar qué } \\
\text { cuestionarios están } \\
\text { actualmente } \\
\text { disponibles para } \\
\text { medir la } \\
\text { satisfacción de las } \\
\text { familias con la } \\
\text { atención en la UCI }\end{array}$ & $\begin{array}{l}\text { Revisión } \\
\text { Sistemática }\end{array}$ & $\begin{array}{l}\text { Se encuentran } 27 \\
\text { cuestionarios } \\
\text { diferentes en los } \\
\text { cuales se destacan: } \\
\text { CCFNI, } \\
\text { SCCMFNA, } \\
\text { CCFSS, FS-ICU. }\end{array}$ & $\begin{array}{c}\text { CCFNI Y FS-ICU, muestran las } \\
\text { mejores propiedades psicométricas } \\
\text { mas investigadas y por lo tanto se } \\
\text { recomienda su uso. }\end{array}$ \\
\hline $\begin{array}{l}\text { Sánchez, } \\
\text { Fernández } \\
\quad \& \\
\text { Fernández } \\
\text { (2016). }\end{array}$ & $\begin{array}{c}\text { Describir las } \\
\text { necesidades de la } \\
\text { familia del paciente } \\
\text { ingresado en la UCI } \\
\text { y la opinión de sus } \\
\text { profesionales sobre } \\
\text { aspectos relativos a } \\
\text { la presencia } \\
\text { familiar. }\end{array}$ & $\begin{array}{c}\text { Estudio } \\
\text { descriptivo } \\
\text { prospectivo }\end{array}$ & $\begin{array}{l}\text { CCFNI, } \\
\text { cuestionario } \\
\text { opiniones de los } \\
\text { profesionales } \\
\text { sanitarios de la } \\
\text { UCI }\end{array}$ & $\begin{array}{c}\text { Familiares } 91,4 \% \text { comprendió } \\
\text { información recibida, } \mathrm{p}<0,05 \text { mostro } \\
\text { conformidad con política de visitas. } \\
\text { Profesionales reclaman formaciones } \\
\text { habilidades comunicativas. }\end{array}$ \\
\hline
\end{tabular}




\begin{tabular}{|c|c|c|c|c|}
\hline $\begin{array}{c}\text { Galvis \& } \\
\text { Salamanca } \\
\text { (2014). }\end{array}$ & $\begin{array}{c}\text { Describir las } \\
\text { necesidades del } \\
\text { cuidador familiar } \\
\text { de pacientes } \\
\text { adultos crónicos } \\
\text { hospitalizados en } \\
\text { UCI en una } \\
\text { institución } \\
\text { prestadora de salud } \\
\text { privada en } \\
\text { Villavicencio, } \\
\text { Colombia. }\end{array}$ & $\begin{array}{l}\text { Descriptivo, } \\
\text { de corte } \\
\text { transversal y } \\
\text { de tipo } \\
\text { cuantitativo. }\end{array}$ & CCFNI & $\begin{array}{c}\text { La necesidad considerada más } \\
\text { importante es seguridad } 86 \% \text {, } \\
\text { información } 82 \% \text {, proximidad } 78 \% \text {. }\end{array}$ \\
\hline $\begin{array}{l}\text { Padilla \& } \\
\text { Rojas } \\
\text { (2018). }\end{array}$ & $\begin{array}{l}\text { Identificar el grado } \\
\text { de importancia de } \\
\text { las necesidades de } \\
\text { los familiares de } \\
\text { pacientes en una } \\
\text { unidad de cuidados } \\
\text { intensivos (UCI). }\end{array}$ & $\begin{array}{c}\text { Estudio } \\
\text { descriptivo, } \\
\text { comparativo } \\
\text { y transversal }\end{array}$ & CCFNI. & $\begin{array}{l}\text { La necesidad mas importante } \\
\text { seguridad, e información, la de menor } \\
\text { importancia se relaciona con } \\
\text { apoyo. }\end{array}$ \\
\hline $\begin{array}{l}\text { Omari } \\
(2009) \text {. }\end{array}$ & $\begin{array}{c}\text { Identificar las } \\
\text { necesidades } \\
\text { autopercibidas de } \\
\text { los jordanos } \\
\text { adultos que tienen } \\
\text { familiares en las } \\
\text { unidades de } \\
\text { cuidados intensivos } \\
\text { y explorar si se } \\
\text { satisfacen esas } \\
\text { necesidades. }\end{array}$ & $\begin{array}{c}\text { Cuantitativo } \\
\text { fue de nivel } \\
\text { descriptivo, } \\
\text { de corte } \\
\text { transversal } \\
\text { prospectivo }\end{array}$ & CCFNI, MNI. & $\begin{array}{l}\text { Las necesidades más importantes } \\
\text { información, y estar seguro. }\end{array}$ \\
\hline $\begin{array}{l}\text { Gómez- } \\
\text { Carretero } \\
\text { et al. } \\
\text { (2009). }\end{array}$ & $\begin{array}{l}\text { Valorar el grado de } \\
\text { satisfacción y } \\
\text { necesidad de } \\
\text { información en } \\
\text { familiares de } \\
\text { pacientes } \\
\text { programados para } \\
\text { una intervención } \\
\text { quirúrgica que } \\
\text { requerirán un } \\
\text { ingreso en UCI, y } \\
\text { su relación con la } \\
\text { depresión y } \\
\text { ansiedad. }\end{array}$ & $\begin{array}{c}\text { Cuantitativo } \\
\text { fue de nivel } \\
\text { descriptivo, } \\
\text { de corte } \\
\text { transversal } \\
\text { prospectivo }\end{array}$ & $\begin{array}{c}\text { Inventario de } \\
\text { depresión de Beck } \\
\text { (BDI). Escala de } \\
\text { Ansiedad Estado } \\
\text { (STAI A/E-A/R) } \\
\text { Encuesta de } \\
\text { Satisfacción ante la } \\
\text { Información } \\
\text { (ESI)Encuesta de } \\
\text { Necesidad de } \\
\text { Información. }\end{array}$ & $\begin{array}{l}\text { Familiares presentaron síntomas } \\
\text { depresivos, y ansiedad, en cuanto a } \\
\text { necesidades la información esta } \\
\text { insatisfecha. }\end{array}$ \\
\hline $\begin{array}{c}\text { Gonçalves } \\
\text { et al., } \\
\text { (2006). }\end{array}$ & $\begin{array}{c}\text { Caracterizar a los } \\
\text { pacientes } \\
\text { ingresados en la } \\
\text { UCI como los datos } \\
\text { bio-sociales y de } \\
\text { hospitalización, y } \\
\text { verificar las } \\
\text { necesidades diarias } \\
\text { de los cuidados de }\end{array}$ & $\begin{array}{l}\text { Descriptivo, } \\
\text { de corte } \\
\text { transversal y } \\
\text { de tipo } \\
\text { cuantitativo }\end{array}$ & $\begin{array}{c}\text { Para la recolección } \\
\text { de datos se utilizó } \\
\text { un documento } \\
\text { titulado } \\
\text { "Evaluación de las } \\
\text { necesidades diarias } \\
\text { de cuidado", } \\
\text { compuesto de } 3 \\
\text { partes: la }\end{array}$ & $\begin{array}{l}\text { Los pacientes con alta prolongada en } \\
\text { UCI reciben menor manejo } \\
\text { terapéutico, mayores gastos y gran } \\
\text { demanda de trabajo para enfermería. }\end{array}$ \\
\hline
\end{tabular}




\section{Cultura de los Cuidados}

\begin{tabular}{|l|c|c|c|c|}
\hline & enfermería de & & identificación del \\
acuerdo con NAS. & & paciente, los datos \\
& & & \\
& & & únicos y NAS, con \\
& & elesglose de los \\
& & componentos & \\
\hline
\end{tabular}

FUENTE: Elaboración propia

TABLA 4: Generalidades de escalas, categorías, validez y características de diseño

\begin{tabular}{|c|c|c|c|c|c|}
\hline \multirow[b]{2}{*}{ Características } & \multicolumn{5}{|c|}{ Escalas para Familiares } \\
\hline & CCFSS & $\begin{array}{c}\text { Family satisfaction } \\
\text { survey }\end{array}$ & SCCMFNA & FS-ICU & CCFNI \\
\hline Nombre & $\begin{array}{l}\text { Critical Care Family } \\
\text { Satisfaction survey }\end{array}$ & $\begin{array}{c}\text { Family satisfaction } \\
\text { survey }\end{array}$ & $\begin{array}{c}\text { Society of Critical } \\
\text { Care Medicine`s } \\
\text { Family } \\
\text { Needs } \\
\text { Assessment }\end{array}$ & $\begin{array}{c}\text { Family Satisfaction } \\
\text { In The Intensive Care } \\
\text { Unit }\end{array}$ & $\begin{array}{c}\text { Critical Care } \\
\text { Family Need Interventory }\end{array}$ \\
\hline № Ítems & 20 & 15 & 14 & 34 & 45 \\
\hline Población & Familia & Familia & Familia & Familia & Familia \\
\hline Puntuación & $\begin{array}{l}5 \text { a } 1 \text { (muy satisfecho, } \\
\text { muy insatisfecho" }\end{array}$ & $\begin{array}{c}1 \text { a } 5 \text { (1 muy escaso, } 5 \mathrm{~m} \\
\text { bien }) .\end{array}$ & $\begin{array}{l}1 \text { a } 4 \text { puntuación } \\
\text { total } 14 \\
\text { satisfactoria, } 56 \\
\text { menos } \\
\text { satisfactoria }\end{array}$ & $\begin{array}{l}\text { Excelente (100), muy } \\
\text { bien }(75) \text {,malo }(0), \mathrm{Si} \\
(100), \mathrm{No}(0)\end{array}$ & $\begin{array}{l}\text { Likert 0-4 0"no } \\
\text { importante) } \\
\text { 4"muy importante" }\end{array}$ \\
\hline Validez & alfa: 0,91 & alfa:0,94 & alfa: 0,76 & alfa: 0,97 & alfa: 0,88 \\
\hline Categorías & $\begin{array}{c}\text { Seguridad, información, } \\
\text { proximidad apoyo } \\
\text { y confort. }\end{array}$ & $\begin{array}{c}\text { Seguridad, informació } \\
\text { apoyo, confort, } \\
\text { cualidades del profesior } \\
\text { satisfacción } \\
\text { con la atención. }\end{array}$ & $\begin{array}{l}\text { Información, } \\
\text { apoyo, confort, } \\
\text { cualidades del } \\
\text { profesional. }\end{array}$ & $\begin{array}{l}\text { Información, confort, } \\
\text { toma de decisiones, } \\
\text { cuidado al paciente, } \\
\text { cualidades del } \\
\text { profesional, } \\
\text { satisfacción general. }\end{array}$ & $\begin{array}{c}\text { Seguridad, información, } \\
\text { proximidad, } \\
\text { apoyo, confort. }\end{array}$ \\
\hline
\end{tabular}


Cultura de los Cuidados

\begin{tabular}{|c|c|c|c|}
\hline \multirow[b]{2}{*}{ Características } & \multicolumn{3}{|c|}{ Escalas para Pacientes y Profesionales } \\
\hline & Care- Q & $\begin{array}{c}\text { Necesidades } \\
\text { Humanas Básic }\end{array}$ & NAS \\
\hline № Ítems & 50 & 4 áreas & 23 \\
\hline Población & Pacientes & Pacientes & Pacientes - Enfermeras \\
\hline Puntuación & Sub-escalas & Sub-escalas & Sub-escalas \\
\hline Validez & alfa: 0,88 & IC: 0,80 & IVC: 0,77 \\
\hline Categorías & $\begin{array}{c}\text { Accesibilidad, facilita y explica, } \\
\text { conforta, se anticipa, mantiene } \\
\text { confianza, monitorea y hace seguimiento. }\end{array}$ & $\begin{array}{l}\text { Identificación, } \\
\text { entrevista, } \\
\text { examen físico, } \\
\text { impresión de } \\
\text { enfermería. }\end{array}$ & $\begin{array}{c}\text { Diario de actividades, soporte ventilatorio, soporte } \\
\text { cardiovascular, } \\
\text { soporte neurológico, soporte metabólico, } \\
\text { intervenciones específicas. }\end{array}$ \\
\hline
\end{tabular}

FUENTE: Elaboración propia

A partir del análisis de los estudios se determinaron tres categorías: Escalas de medición de necesidades de la familia en UCI, escalas de medición de necesidades de pacientes críticos adultos y escalas de medición de necesidades de los profesionales que laboran en la UCI.

\section{Escalas de medición de necesidades de la familia en UCI}

Existe en la literatura reportada alrededor de 27 instrumentos de medición, los más utilizados y con mayores estándares de calidad dentro de la práctica clínica; CCFNI, FS-ICU SCCMFNA, FAMILY, CCFSS, NMI, cada uno de estos instrumentos cuenta con varias versiones a nivel mundial, donde se evalúa ampliamente su calidad psicométrica, siendo el CCFNI con un alfa de Cronbach de 0,88 el que muestra mejor adaptación transcultural y es el más utilizado, seguido del cuestionario FS-ICU que reporta un alfa de Cronbach de 0,97 (Olano \& Vivar, 2012; Gómez, Ballester \& Gil, 2011; Ban et al. 2015; Sánchez, Fernández \& Fernández, 2016; Galvis \& Salamanca, 2014; Padilla \& Rojas, 2018; Omari, 2009;
Gómez-Carretero et al., 2009).

\section{Escalas de medición de necesidades de pacientes críticos adultos}

La literatura descrita aporta 2 instrumentos de medición; escala $C A R E-Q$ y Necesidades Humanas Básicas (Pedrechi \& Camargo, 2015; Sepúlveda, Cardenas \& Rojas, 2009; Ramalho et al., 2013). El Instrumento CARE-Q es el más utilizado a nivel mundial en diferentes contextos. En Colombia el instrumento mostró un alfa de Cronbach de 0,88 (Sepúlveda, Cardenas \& Rojas, 2009). Por otro lado, el Instrumento de Necesidades Humanas Básicas se construyó a partir de identificar las características especiales de los pacientes de la UCI en especial a la condición fluctuante de salud, medios invasivos, entre otros, este cuestionario muestra alto indica de confiabilidad IC > 0,80 (Ramalho et al., 2013).

\section{Escalas de medición de necesidades de los profesionales que laboran en la UCI}

Los instrumentos de medición en la categoría de necesidades para profesionales de la salud UCI son las escalas NAS, TISS y 
se centran en la carga laboral (Gonçalves et al., 2006). No existe evidencia de instrumentos validados para conocer necesidades de otra índole de los profesionales, la única evidencia disponible actualmente es un cuestionario $A D H O C$ adaptado a la población, sin embargo, esta evalúa la percepción del profesional frente a las necesidades del familiar (Sánchez et al., 2016).

\section{DISCUSIÓN}

En el entorno de la UCI identificar las necesidades de cuidado en pacientes $\mathrm{y}$ cuidadores, incluyendo al equipo de salud como cuidadores temporales, se considera como un componente clave para el mejoramiento del cuidado al final de la vida (Breen et al., 2001; Carlet et al., 2004). En la 2002, la Organización Mundial de la Salud define los cuidados paliativos como «el enfoque que mejora la calidad de vida de pacientes y familias que se enfrentan a los problemas asociados con enfermedades amenazantes para la vida, a través de la prevención y alivio del sufrimiento, por medio de la identificación temprana y la impecable evaluación y tratamiento del dolor y otros problemas físicos, psicosociales y espirituales».

Con relación a los instrumentos de evaluación disponibles en la literatura, se evidencia que la mayoría de escalas validadas están dirigidas a evaluar las necesidades de los familiares en la UCI, lo cual resalta la importancia de los cuidadores en la atención al final de la vida. Los instrumentos CCFSS, y el FS-ICU reportan mejores características psicométricas $\mathrm{y}$ utilidad clínica; dentro de las similitudes ambas escalas miden confort e información, las diferencias parten en relación al fenómeno a medir; CCFNI valora las necesidades y FS-ICU la satisfacción. Aunque estos son fenómenos diferentes se ha identificado que su aplicación combinada es de gran valor para una mejor comprensión de las necesidades percibidas (Broek, 2015; Heiland, 2002).

Respecto a los resultados de los cuestionaros de medición de las necesidades de pacientes de la UCI la evidencia no es amplia, debido a la complejidad de los pacientes en estado crítico que dificulta la aplicación de instrumentos en la práctica clínica (Enciso, 2015; Pardavila, 2011). Se identificaron 2 cuestionarios, Necesidades Humanas Básicas y el cuestionario Care- $Q$ con características psicométricas apropiadas que se pueden aplicar conjuntamente para la valoración de las necesidades del paciente crítico (Pedrechi \& Camargo, 2015; Sepúlveda, Cárdenas \& Rojas, 2009; Ramalho et al., 2013; Sepúlveda, Cárdenas \& Rojas, 2009).

Por otro lado, los resultados de la revisión reafirman la necesidad de concebir al profesional que labora en la UCI como sujeto de cuidado. Es insípida la producción científica en este ámbito, aunque existen evidencias que demuestran que la sobrecarga emocional del profesional se complejiza por los conflictos éticos que son prevalentes en el manejo del paciente grave, y están asociados habitualmente al desacuerdo sobre decisiones terapéuticas entre el paciente y familia con los clínicos, a la omisión y retirada del tratamiento de soporte vital, y a las decisiones de representación, influyendo así en la interacción entre profesionales y familiares y en las medidas tendientes a decidir el mejor manejo del paciente al final de la vida (Camargo, 2015). Por tanto, valorar sus necesidades permitirá disminuir y tratar oportunamente casos de síndrome de Bournot caracterizado por agotamiento emocional, 
despersonalización y baja autoestima (Grupo de Certificación De Proyecto HU-CI, 2017).

El profesional de la salud como sujeto multidimensional tiene diversas necesidades (Hernandez-Zambrano et al., 2016), sin embargo, la literatura descrita solo aporta cuestionarios desde el punto de vista de carga laboral, convirtiéndolas automáticamente en escalas para pacientes, ya que permiten distribuir los cuidados de acuerdo a la complejidad y características de ellos. Este fenómeno genera que el personal de salud presente dificultades en la relación terapéutica con pacientes y familiares, relacionadas con el distanciamiento emocional (Velarde et al., 2017) y pone de manifiesto que la disciplina de enfermería ha investigado acerca de las necesidades de pacientes y familia, despersonalizándose de su propio cuidado.

Respecto a las dimensiones que evalúan las escalas, son predominantes los dominios de seguridad, información, proximidad, apoyo, confort y soporte de síntomas físicos. Sin embargo, es insípida la valoración de necesidades psicosociales y espirituales que son esenciales en la atención al final de la vida. Este fenómeno puede obedecer al modelo de gestión de la muerte en la UCI que se centra en el modelo biomédico y excluye la percepción de las enfermeras, familiares y pacientes como sujetos muldimensionales (Gálvez et al., 2011). En esta línea, la investigación de Santos et al., (2017), explica la importancia de evaluar además del estado clínico, el contexto socioeconómico $y$, principalmente, espiritual de las personas dado que sus expectativas, sus deseos y anhelos deben ser respetados en el momento de la muerte.

Dentro de las limitaciones del estudio se encontró la falta de información respecto a la sensibilidad y utilidad de las distintas escalas, además son escasos los estudios que describen las características psicométricas de forma detallada. También es importante considerar la adaptación transcultural de los instrumentos, como componente imprescindible para su aplicación en diferentes contextos socio-culturales. Por otro lado, los artículos se recuperaron desde la plataforma de la Fundación Universitaria de Ciencias de la salud, factor que incluye en la muestra bibliográfica.

\section{CONCLUSIONES}

Los sujetos que conviven en la UCI, tienen necesidades derivadas del sufrimiento que les ocasiona el enfrentarse a la muerte. Los resultados de la revisión ponen de manifiesto la necesidad de desarrollar y validar instrumentos que evalúen las necesidades psicosociales y espirituales de pacientes, familia y profesionales en el contexto de la UCI, dado que las escalas validadas y utilizadas en este ámbito se focalizan en la valoración de soporte de síntomas físicos y satisfacción.

Son escasas las evidencias de instrumentos que evalúen las necesidades de los profesionales que laboran en la UCI, justificando la necesidad de plantear una línea de investigación que genere conocimiento en este ámbito.

Las implicaciones de estos resultados para favorecer una buena muerte en la UCI son significativas, dado que la evaluación de las necesidades es un imperativo ético para mejorar los cuidados al final de la vida del paciente crítico, de sus familiares y de los profesionales de la salud

\section{BIBLIOGRAFÍA}

- Bagshaw, S. M., Webb, S. A., Delaney, A., George, C., Pilcher, D., Hart, G. K. \& Bellomo, R. (2009). Very old patients admitted to intensive care in Australia and 
New Zealand: a multi-centre cohort analysis. Critical Care, 13(2), R45.

- Ballester, R., Gil, B., Gil, M., \& Gómez S. (2011). Afrontamiento de la muerte en familiares de pacientes ingresados en una unidad de cuidados intensivos: valoración diferencial en función de variables sociodemográficas. Med Paliat, 18(2), 46-53.

- Ban, J., Brunsveld, A., Zedlitz, A., Girbes, A., Jonge, E. \& Arbous, M. (2015). Questionnaires on Family Satisfaction in the Adult ICU: A Systematic Review Including Psychometric Properties. Critical Care Medicine, 43(8), 1731-44.

- Breen, C., Abernethy, A., Abbott, K., \& Tulsky, J. (2001). Conflict associated with decisions to limit lifesustaining treatment in intensive care units. J Gen Intern Med, 16, 283 - 9.

- Broek, J., Brunsveld, A., \& Zeediltz, A. (2015). Questionnaires on Family Satisfaction in the Adult ICU: A Systematic Review Including Psychometric Properties. Crit Care Med, 43(8), 1731-44.

- Camargo, R.D. (2015). El enfermo crónico en la fase final de su enfermedad en cuidado intensivo requiere transición del cuidado curativo al cuidado paliativo. Acta Colomb Cuid Intensivo, 15(1), 45-48.

- Carlet, J., Thijs, L., Antonelli, M., Cassel, J., Cox, P., Hill, N. et al. (2004). Challenges in end-of-life care in the ICU. Intensive Care Med, 30, 770-84.

- Enciso, C. (2015). El paciente crítico, una familia enferma. Descripción de la experiencia de la familia de pacientes adultos hospitalizados en cuidado intensivo, a través de la teoría de la intersubjetividad. (Tesis de maestría no publicada) Universidad del Bosque: Bogotá.

- Gálvez González, M., Ríos Gallego, F., Fernández Vargas, L., Águila Hidalgo, B., Muñumel Alameda, G. \& Fernández Luque, C. (2011). El final de la vida en la Unidad de Cuidados Intensivos desde la perspectiva enfermera: un estudio fenomenológico. Enfermería Intensiva, 22(1), 13-21.

- Galvis, C. \& Salamanca, E. (2014). Perceived Needs in Family Caregivers of Adults Hospitalized in an Intensive Care Unit of a Private Health Providing Institution in Villavicencio, Colombia. Investig. Enferm. Imagen Desarr, (2), 81-94.

- Gómez, S., Ballester, R. \& Gil, B. (2011). El Cuestionario de Necesidades de los Familiares de Pacientes de Cuidados Intensivos (CCFNI) versión breve: adaptación y validación en población española. An. Sist. Sanit. Navar. 34 (3), 349-361.

- Gómez-Carretero, P., Soriano, J. F., Monsalve, D.V. \& Andrés J. (2009). Satisfacción con la Información: Posible Variable Interviniente en el Estado de Ánimo de Cuidadores Primarios de Pacientes Críticos. Clínica y Salud, 20(1), 91-105.

- Gonçalves, L. A, Garcia, P.C, Toffoleto, M. C, Telles, S. C. R \& Padilha, K. G. (2006). Necessidades de cuidados de enfermagem em Terapia Intensiva: evolução diária dos pacientes segundo o Nursing Activities Score (NAS). Revista Brasileira de Enfermagem, 59(1), 56-60.

- Grupo de Certificación De Proyecto HU-CI. (2017). Manual de buenas prácticas de humanización en UCI. Madrid: Proyecto HU-CI.

- Gutiérrez, B., Blanco, J., Alvariño, A., Luque, M., \& Ramírez, M. (2008). Experiencias Percepciones y Necesidades en la UCI: Revisión Integradora. Enfermería global, 12. Recuperado de http://revistas.um.es/eglobal/article/view/822/842.

- Hernández-Zambrano, S.M., Hueso-Montoro, C., Montoya-Juárez, R., Gómez-Urquiza, J.L. \& Bonillde-las-Nieves, C. (2016). Metaestudio cualitativo sobre vivencias y gestión del cotidiano en adultos mayores que padecen enfermedades crónicas. Cultura de los Cuidados, 20(44), 75-90.

- Jimeno, L., López, P., Ezenarro, M., Margall, C., \& Asiain, E. (2007). Cuidados del paciente al final de la vida: ayudas y obstáculos que perciben las enfermeras de Cuidados Intensivos. Enferm Intensiva, 18(1), 3-14.

- Marin, M., Hernandez, A., Rubio, O., Monzon, J.L. \& Cabré, L. (2016). Pacientes críticos al final de la vida: estudio multicéntrico en Unidades de Cuidados Intensivos españolas. Med Intensiva, 40, 448-50.

- Olano, M. \& Vivar, G. (2012). Instruments for the assessment of the needs of family members of patients in intensive care units: a systematic review. An. Sist. Sanit. Navar, 30(1), 53-67.

- Omari, F. (2009). Perceived and Unmet Needs of Adult Jordanian Family Members of Patients in ICU. J Nurs Scholarsh, 41(1), 28-34.

- Organización Mundial de la Salud. (2002). Cuidados paliativos [Sede web]. Recuperado de http://www.who.int/cancer/palliative/es/.

- Padilla, C. \& Rojas, N. (2018). Necesidades de los familiares de pacientes críticos en un hospital académico de Chile. Enfermería Intensiva, 29(1), 32-40.

- Pedrechi, D. \& Camargo, I. (2015). Percepción de las enfermeras y pacientes, del cuidado brindado por el profesional de enfermería, en la unidad de cuidados intensivos del Hospital Regional. Rev. Cien de Enfermería, 17, 17.

- Ramalho, J. M., Fontes, W. D. \& Nóbrega, M. M. L. (2013). Instrumento de coleta de dados de enfermagem em Unidade de Terapia Intensiva Geral. Revista Brasileira de Enfermagem, 66(4), 535-542.

- Santos, R. P., Dias, P. F., Ribeiro, J. H. M., Mendes, M. A., \& Silva, J.V. (2017). Reflexiones sobre el escenario de la muerte en la perspectiva paliativa. Cultura de los Cuidados (Edición digital), 21(49). Recuperado de http://dx.doi.org/10.14198/cuid.2017.49.18.

- Sánchez, R. \& Echeverry, J. (2004). Validación de 


\section{Cultura de los Cuidados}

escalas de medición en salud. Rev. Salud pública, 6 (3), 302-318.

- Sánchez, V., Fernández, P. \& Fernández, M. (2016). Análisis de las necesidades de la familia del paciente crítico y la opinión de los profesionales de la unidad de cuidados intensivos. Medicina Intensiva, 40(9), 527540 .

- Sepúlveda, G., Cárdenas, O. \& Rojas, L. (2009). Estudio piloto de la validación del cuestionario 'CARE-Q' en versión al español en población colombiana. Rev col enfermería (Edición digital), IV(4). Recuperado

de http://www.imbiomed.com.mx/1/1/articulos.php?m ethod=print\&id_revista $=245 \&$ id_seccion $=4200 \& i d \_$ej emplar $=6980 \&$ id articulo $=69884$.

- Silva, R.S., Pereira, A., Ribeiro, A., Marinho, C., Carvalho, I. \& Ribeiro, R. (2012). Elaboração de um instrumento para coleta de dados de paciente crítico: histórico de enfermagem. Rev. enferm. UERJ, 20(2),
267-73.

- Sprung, C.L., Cohen, S.L., Sjokvist, P., Baras, M., Bulow, H.H., Hovilehto S, et al. (2003). End-of-Life Practices in European Intensive Care Units. The Ethicus Study. J Am Med Assoc, 290, 790-7.

- Velarde, F., García, R., González, R., González, S., Cervantes, B., Álvarez, E., et al. (2017). End of life care difficulties in intensive care units. The nurses' perspective. Gaceta Sanitaria, 31(4), 299-304.

- Vicensi, C. (2016). Reflexión sobre la muerte y el morir en la UCI a partir de la perspectiva profesional en cuidados intensivos. Revista Bioética, 24(1), 64-72.

- Zambrano, G. (2008). Estresores en las unidades de cuidado intensivo. AQUICHAN (Edición digital), 6(1). Recuperado de http://aquichan.unisabana.edu.co/index.php/aquicha n/article/view/89/183. 